\title{
Pitfalls in diagnosis and management of carbon monoxide poisoning
}

\author{
B Roy, R Crawford
}

\begin{abstract}
Five members of one family suffering from carbon monoxide poisoning are described. Three were initially diagnosed as food poisoning cases at another hospital. A high level of suspicion is required to ensure early diagnosis. Indications for hyperbaric oxygen include: loss of consciousness, neurological signs and symptoms other than mild headache, cardiac complications, carboxyhaemoglobin $>\mathbf{4 0} \%$, and pregnancy.

( $f$ Accid Emerg Med 1996;9:62-63)
\end{abstract}

Key terms: carbon monoxide poisoning; diagnosis; treatment; hyperbaric oxygen

Carbon monoxide poisoning is still an important cause of death in the United Kingdom and abroad. ${ }^{1}$ The problem has recently been highlighted in both radio and television broadcasts. ${ }^{23}$ In addition, carbon monoxide poisoning is still underdiagnosed. ${ }^{4}$ Once diagnosed, treatment may be inadequate, particularly with respect to the underuse of hyperbaric oxygen. ${ }^{5}$ We describe an incident affecting five members of a family and review some important potential pitfalls in the diagnosis and treatment of this condition.

\section{Case reports}

A family of five were found collapsed and semiconscious in various rooms throughout their bungalow. They had all eaten chicken the previous evening and complained of nausea, vomiting, dizziness, and headache since then. In addition, both parents had suffered from diarrhoea. Both daughters were taken to the accident and emergency department at Glasgow Royal Infirmary. The remainder of the family were taken to a district general hospital nearer their home.

At the district general hospital, an initial diagnosis of food poisoning had been made. Arrangements were underway for transfer of two cases (2 and 5) to the regional infectious diseases unit. At Glasgow Royal Infirmary, our initial diagnosis was that of acute carbon monoxide poisoning. A prompt diagnosis was made because there was a high level of awareness within the department following previous experience of the condition, and because the subject had recently been reviewed at a departmental meeting. Oxygen treatment was given using a trauma mask with a reservoir bag at a rate of 15 litres per minute. The initial carboxyhaemoglobin $(\mathrm{COHb})$ concentrations confirmed the diagnosis and are listed in the $\mathbb{\mathscr { D }}$ table. While the results were awaited, the o condition of both daughters improved, with a decrease in nausea and headache and an increase in their Glasgow Coma Scale from an initial $14(E=3)$ to $15(E=4)$.

The other hospital was contacted and the diagnosis in the other patients was confirmed. The carboxyhaemoglobin concentrations and clinical features of all five patients are shown in the table.

We consulted the Hyperbaric Oxygen Unit at Aberdeen Royal Infirmary and treatment with hyperbaric oxygen was recommended for all five family members. This information was conveyed to the hospital dealing with the other $₹$ family members, but was met with initial $\vec{\bullet}$ reluctance to transfer. All five were eventually flown to Aberdeen, where they received hyperbaric oxygen at $2.5 \mathrm{~atm}$ for $1 \mathrm{~h}$ and 2 atm for $2 \mathrm{~h}$.

Two months after the episode, all family members were symptomless, though their condition was not formally assessed. The Health and Safety Executive believed the source of poisoning to be the gas central heating, due to a downdraft in the flue.

\section{Discussion}

The index of suspicion for carbon monoxide poisoning must be high. It is important to note that several people may be affected simultaneously and that symptoms are often nonspecific: nausea, vomiting, dizziness, headache, 은 and even diarrhoea. Both the above may lead to a misdiagnosis ${ }^{6}$ of, for example, "flu" or 을 "gastroenteritis".

Carboxyhaemoglobin levels greater than $10 \%$ O indicate poisoning. The values are, however, $\stackrel{\sim}{\mathcal{O}}$ time dependent ${ }^{7}$ and are influenced by oxygen treatment given before assessment. In addition, symptoms correlate poorly with the carboxyhaemoglobin concentration, especially with $\overparen{\varnothing}$ chronic low level exposure, and the decision to treat with hyperbaric oxygen must be made on clinical grounds. This is because the toxicity is probably mediated through cellular respiration,

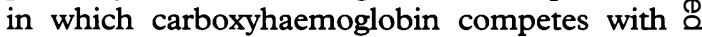
oxygen for cytochrome a3, rather than simply by its combination with haemoglobin leading to a decrease in available oxygen. ${ }^{8}$

There is a high incidence of late neuropsychiatric sequelae in patients who have $\stackrel{ }{\rightleftharpoons}$ suffered severe carbon monoxide poisoning. This may be reduced by treatment with 
Carboxyhaemoglobin levels and clinical features in five patients with acute carbon monoxide poisoning.

\begin{tabular}{lllll}
\hline Case No & Age & Sex & $\begin{array}{c}\text { Initial COHb } \\
(\%)\end{array}$ & Clinical features \\
\hline 1 & 47 & $\mathrm{M}$ & $23 \%$ & nausea, vomiting, headache, diarrhoea \\
2 & 40 & $\mathrm{~F}$ & $30 \%$ & nausea, vomiting, headache, diarrhoea \\
3 & 14 & $\mathrm{~F}$ & $24 \%$ & nausea, vomiting, headache \\
4 & 12 & $\mathrm{~F}$ & $40 \%$ & nausea, vomiting, headache \\
5 & 10 & $\mathrm{M}$ & $16 \%$ & nausea, vomiting, headache \\
\hline
\end{tabular}

hyperbaric oxygen even when it is given late. ${ }^{9}$ Chronic low level exposure is believed to be prevalent in the community and the diagnosis is usually missed because of the protean manifestations. ${ }^{10}$

It is worth noting that the presence of carboxyhaemoglobin makes monitoring by pulse oximetry unreliable because the displayed saturation level equals the sum of oxyhaemoglobin and carboxyhaemoglobin. ${ }^{11}$ Significant hypoxia may therefore be masked and the clinician lulled into a false sense of security.

Initial treatment should be with a nonrebreathing circuit with a non-return valve, oxygen reservoir, and tight fitting mask. The type of mask used by ourselves only delivers a maximum inspired oxygen concentration of $85 \%$ if applied correctly and connected to high flow oxygen. It is important to supply the highest oxygen concentration possible as the elimination half life of carbon monoxide is 240 minutes when breathing air, but only 60 minutes when breathing $100 \%$ oxygen. ${ }^{7}$

The initial reluctance to transfer the other three patients suggests that the indications and potential benefits of hyperbaric oxygen are not well enough known; the reluctance appeared to be based on the belief that there is little scientific evidence to support the use of hyperbaric oxygen in carbon monoxide poisoning, as indicated by a recent survey by the BMA's Board of Science and Education. ${ }^{5}$ The indications for the use of hyperbaric oxygen following carbon monoxide poisoning are:
- Loss of consciousness at any time

- Neurological or psychiatric symptoms or signs, other than a mild headache, identified on thorough testing

- Cardiac complications such as arrhythmias, ischaemic chest pain, or ischaemic ECG changes

- Carboxyhaemoglobin concentration more than $40 \%$, regardless of symptoms

- Pregnancy

Although all of these indications are well described in published reports, we feel that they require stressing as they may often be overlooked. In addition, we recommend that carbon monoxide poisoning be routinely excluded in all cases of suspected food poisoning or sudden illness affecting several people, especially family members, who are brought from the same location.

The authors would like to thank Dr J Ross, FCANAES, Senior Lecturer, Environmental and Occupational Medicine, University of Aberdeen, for providing information about the five patients in the case report.

1 Broome JR, Pearson RR, Skrine H. Carbon monoxide poisoning: forgotten not gone! $B r \mathcal{F}$ Hosp Med 1988;39: 298-305.

2 Radio Four, 9 February 1994: "Face the Facts".

3 BBC 1, 7 March 1994: "Watchdog".

4 Myres RAM, Goldman B. Planning an effective strategy for carbon monoxide poisoning. Emerg Med Rep 1987;8 carbon m-200.

5 BMA Board of Science and Education. Clinical hyperbaric medicine facilities in the UK. London: British Medical medicine facilities in

6 Grace TW, Platt FW. Subacute carbon monoxide poisoning. Another great imitator. $\mathscr{f} A M A$ 1981;246: 1698-700.

7 Piantadosi CA. Carbon monoxide, oxygen transport, and oxygen metabolism. F Hyperbaric Med 1987;2:27-44.

8 Goldbaum LR, Ramirez RG, Absalon KB. What is the mechanism of carbon monoxide toxicity? Aviat Space Environ Med 1975;46:1289.

9 Smith JS, Brandon S. Morbidity from acute carbon monoxide poisoning at 3 year follow up. $B M \mp$ 1973; 318-21.

10 Crawford R, Campbell DGD, Ross J. Carbon monoxide poisoning in the home: recognition and treatment. $B M \mathcal{J}$ poisoning in the

11 Vegfors $M$, Hennmarket $C$. Carboxyhaemoglobin and pulse oximetry. Br f Anaesth 1991;66:625-6.

Accident and
Emergency
Department,
Leeds General
Infirmary,
Great George Street,
Leeds LS1 3EX
A R Wass
J P Sloan
Correspondence to:
A R Wass,
Senior Registrar in Accident

\title{
Cortical bone cyst following a greenstick radial fracture
}

\author{
A R Wass, J P Sloan
}

\begin{abstract}
There are only a few reports of well defined cystic lesions of the peripheral skeleton following fracture. In children, these lesions are mostly small cortical defects affecting the distal radial metaphysis after a greenstick or torus fracture. A cyst is reported complicating a greenstick fracture, together with a brief review of published reports confirming that these are asymptomatic lesions which can be managed conservatively without further investigation.
\end{abstract}

(f Accid Emerg Med 1996;13:63-64)
Key terms: cortical bone cyst; greenstick fracture.

\section{Case report}

A 9 year old girl presented with a painful left wrist after a fall. Ten months previously she had fractured the distal end of the left radius (fig 1) and had made a full functional recovery. Clinical examination suggested further bony injury but radiographs revealed a cystic lesion in the distal radius, adjacent to the healed fracture (fig 2). A bone scan was "cold" at the site of the cyst. The symptoms settled quickly and the patient remained asymptomatic at follow up. 